\title{
Éléments pour une biographie
}

Elemente für eine Biographie

Elements for a biography

\section{Manuel Durand-Barthez}

\section{OpenEdition}

\section{Journals}

Édition électronique

URL : https://journals.openedition.org/ceg/8905

DOI : $10.4000 /$ ceg. 8905

ISSN : 2605-8359

\section{Éditeur}

Presses Universitaires de Provence

Édition imprimée

Date de publication : 1 mai 2013

Pagination : 279-284

ISBN : 0751-4239

ISSN : 0751-4239

\section{Référence électronique}

Manuel Durand-Barthez, «Éléments pour une biographie », Cahiers d'Études Germaniques [En ligne], 64 2013, mis en ligne le 02 janvier 2020, consulté le 02 juin 2021. URL : http://journals.openedition.org/ ceg/8905; DOI : https://doi.org/10.4000/ceg.8905 


\section{Éléments pour une biographie Manuel DURAND-BARTHEZ \\ École nationale des Chartes}

Née à Hamburg en 1938, Ortrud Beginnen a exercé une carrière de comédienne, tantôt chanteuse de cabaret, tantôt " diseuse » sur ce que l'on est convenu d'appeler Brettlbühne', tantôt, mais bien plus rarement car, malgré le talent incontestable qu'elle pouvait déployer sur des scènes conventionnelles, le cabaret et le café-théâtre restaient son univers de prédilection, tantôt donc, comédienne plus classique dans des pièces de Shakespeare, de Lessing ou de Brecht.

En seconde partie de cette étude figure le témoignage et l'analyse proposés par James Lyons, metteur en scène et auteur originaire des ÉtatsUnis ; il travaille en Allemagne depuis environ vingt-cinq ans. Il fut le compagnon d'Ortrud Beginnen pendant une quinzaine d'années jusqu'à la mort de celle-ci, enlevée prématurément en 1999 par une longue maladie qui ne l'a pas empêchée de continuer à jouer avec brio et détermination jusqu'aux derniers jours.

J. Lyons met l'accent sur les différentes étapes qui jalonnèrent la carrière de l'artiste à compter du milieu de la décennie 1960. Pour notre part, nous souhaitons dans un premier temps présenter Ortrud Beginnen, telle qu'elle s'est révélée dans son autobiographie intitulée Guck mal, schielt ja! publiée en $1975^{2}$.

\section{Les aïeules maudites : l'héritage}

Le destin d'Ortrud Beginnen s'est forgé en réaction à de multiples déconvenues (et le mot est bien faible) subies par la plupart des femmes de sa

1 Terme difficilement traduisible, ćvoquant un mélange de scèncs de Commedia dell'Arte, de café-théâtre ct de chansonniers.

2 Ortrud BEGINNEN, Guck mal, schielt ja! Manuskripte aus dem Katastrophenkoffer («Voyez done ! Elle louche! Manuserits extraits de la boîte à catastrophes »), C. Bertelsmann Verlag, 1975. 
lignée depuis trois générations. Deux d'entre elles furent poussées au suicide par la pression et la convention sociales. On ne peut comprendre la personnalité que révèlent ses spectacles sans évoquer le parcours de ces femmes depuis le début du siècle dernier. Ce que l'artiste nommera la lignée des Duncker/Böttcher/Beginnen, subit au fil des ans une kyrielle d'événements singulièrement attentatoires, tant à l'encontre de leur vie au sens propre qu'à celle de leur dignité.

Frieda Duncker, cantinière de son état, séduite par un trompettiste de fanfare en 1904, est livrée à la vindicte de sa famille, de sa mère Lina en particulier. Celle-ci propose sa fille en mariage au coupable, qui lui signifie vertement son refus catégorique d'épouser une fille qui n'est plus vierge. Le corps de Frieda est retrouvé peu après dans le canal de l'Elde à Lübz, petite bourgade du Mecklenburg.

Lina Duncker, employée au buffet de la gare de Hamburg-Altona, est convaincue du vol d'une demi-livre de beurre sur son lieu de travail en janvier 1919, puis citée à comparaître au tribunal pour répondre de son forfait. Incapable d'assumer la honte que ce délit implique, elle se noie dans l'Isebek-Kanal.

Dans la lignée colatérale des Böttcher, Auguste Viktoria est l'arrièregrand-mère maternelle d'Ortrud Beginnen. Domestique, elle est violentée par un majordome et affligée d'une "grossesse illégitime »". Cette expression revient comme un leitmotiv dans le récit de son arrière-petite-fille.

Auguste Viktoria entretient vis-à-vis de sa mère Clara une relation toujours conflictuelle qui contraint celle-ci à répudier sa fille jugée trop " volage ", un qualificatif qui caractérise sans nuances et à plusieurs reprises le comportement de la plupart de ces femmes, y compris d'Ortrud Beginnen. Auguste Viktoria joue de la trompette, ce même instrument qui, par le biais du coupable Musikmeister Joachim, porta en d'autres temps malheur à Frieda.

Hermine Minna Auguste, fille d'Auguste Viktoria, s'illustre dans son jeune âge lors d'un concours de tir au pigeon à Lübz. Clairement gagnante en regard des règles du jeu, Hermine est proclamée deuxième (et donc à maints égards perdante), car née hors mariage (voir plus haut). Elle est employée de maison dans une famille bourgeoise de Lübz, et victime de la médisance d'Emma, redoutable chef cuisinière. Elle doit quitter cette place et sa mère Augusta l'emmène à Hambourg. De là date l'ancrage de la famille d'Ortrud Beginnen dans la métropole hanséatique.

Quoique malmenée à nouveau dans ses fonction ancillaires, Hermine se permet quelques libertés dont une échappée vers un bal. C'est là qu'elle rencontre Jakob Egidius, emballeur de bananes chez Hapag-Lloyd. Son père, Jacob Heinrich Begien, paysan actif dans la région de Maastricht, était d'origine belge. Son patronyme fut - sinon germanisé, au moins dépouillé de sa consonance flamande - transformé en Beginnen. L'union de Jakob et Hermine était exceptionnelle : « Et ainsi Hermine Böttcher épousa-t-elle

3 O. BEGINNEN, op. cit., p. 24. 
Jacob Egidius Beginnen en tout bien tout honneur, sans être enceinte. Elle incarnait de la sorte cette exception que j'évoquais, parmi les femmes de la lignée Böttcher/Beginnen. ${ }^{4}$. Ortrud Beginnen souligne ce trait avec une acuité particulière.

La voix de Jakob enfant était particulièrement remarquée dans les chorales religieuses ; il fut mélomane toute sa vie, passion qu'il fit partager à ses deux filles : Ingeborg et Gerda. La première se distinguait au piano, la seconde au violon et toutes deux chantaient, en amateur comme leur père, dans un répertoire populaire où figuraient notamment Leo Fall, Hermann Löns et Paul Abraham.

Ingeborg Beginnen vivait, à l'instar des autres membres de sa famille, d'un revenu fort modeste, comme téléphoniste dans une aciérie. Elle donna naissance à Ortrud en 1938. Ingeborg néanmoins, à l'inverse de sa mère, ne sut échapper à la tradition de la « grossesse illégitime ». Elle fut contrainte de déménager, de dissimuler l'événement autant que faire se pouvait, au grand dam des parents, du père en particulier.

Le récit d'Ortrud Beginnen constitue à maints égards un documentaire sociologiquement important sur le quotidien des Allemands à la veille de la Seconde Guerre mondiale et pendant le conflit. Ingeborg Beginnen chante Wagner à l'Opéra de Lille occupée par les Nazis puis revient en Allemagne « exilée thuringeoise de l'intérieur» pour travailler avec sa fille encore jeune dans une ferme à Innien, près de Heide in Holstein, en mars 1946. Les conditions de vie sont là particulièrement difficiles. Les souvenirs d'Ingeborg, autrefois soliste dans la chorale de Sankt-Petri à Hambourg ainsi qu'au Stadtheater Elbing (Prusse orientale) marquent Ortrud, tout comme les talents musicaux du grand-père Jakob.

\section{La naissance difficile de talents contrariés}

Dès sa prime enfance, la nature a contrarié Ortrud Beginnen. Ou plus exactement et de façon plus banale, la pression sociale lui a infligé un sentiment d'illégitimité en matière de goûts et d'envies. Il n'est pas obligatoirement déplacé d'imaginer que l'absence de légitimation qui a empreint sa lignée, féminine en particulier, entacha de manière significative son destin d'artiste, qu'elle dut forger avec obstination jusqu'à sa reconnaissance à Berlin dans les années soixante.

Sans être aucunement disgracieux, le physique d'Ortrud Beginnen enfant puis adolescente déconcertait quelque peu. Elle ne consacre pas moins d'une trentaine de pages au récit d'événements lors desquels elle se trouvait en situation de «représentation », à l'occasion de spectacles scolaires ou de

4 O. BEGINNEN, op. cit., p. 58 (« Und so heiratete Hermine Böttcher Jacob Egidius Beginnen ganz in Ehre, ohne schwanger zu sein. Sie war die Ausnahme, von der ich gesprochen habe, unter den Frauen der Linie Böttcher/Beginnen »). 
cérémonies religieuses. Tout semble s'acharner contre elle. À commencer par ce fameux Silberblick (strabisme) qu'elle commente sur un ton plaisant: «C'est bien par pure coquetterie que ce livre est intitulé 'Voyez donc ! elle louche!' ; car j'ai de fait parfaitement réadapté ma vue depuis lors. » ${ }^{5}$ Les critiques les plus acerbes, parfois vulgaires, sur son apparence sont ressenties comme une profonde blessure. Sa taille excessivement grande pour son âge, son aspect anémié qui dissimule sans doute un mal-être associé à son milieu hostile ; une couleur de cheveux «indéfinissable » et une manière de se coiffer " à la diable "; un nez qui ne lui sied pas, des problèmes dentaires qui l'obligent à rembourser 3500 marks sur deux ans dans un contexte économique difficile; et pour finir, une voix inclassable qui ne la prédispose pas à suivre la tradition familiale. Un chef de chœur de Neumünster dans le Schlewig-Holstein, affirme péremptoirement qu'elle n'a pas de voix. Elle joue en 1951 le rôle du Struwelpeter, «Pierre l'Ébouriffé », l'enfant turbulent et désobéissant : le rôle lui convient à merveille. Ses doigts sont affublés de prolongations griffues redoutables; elle est en outre maquillée de façon exagérée. Dans l'assistance, un boulanger peu discret fait observer à sa petite fille que seules les prostituées se défigurent ainsi. Le réalisme de sa prestation fut remarqué par le Holsteinischer Courier, un véritable événement pour une enfant de cet âge. On interrogea même sa grand-mère pour vérifier que cet enfant était réellement sa petite-fille.

Une adolescence difficile la confronte assez tôt au marché du travail où elle exécute diverses tâches à l'insatisfaction quasi générale de ses employeurs successifs. À vingt-trois ans, elle quitte l'environnement familial pour se rendre à Berlin. Dans ses bagages, réduits à l'extrême, elle emporte les œuvres complètes de Heine. Elle obtient un emploi à la galerie Camilla Speth de la librairie Elwert \& Meurer du Kurfürstendamm. Elle y rencontre de jeunes musiciens, dont un guitariste qui lui propose de chanter sur des textes de Tucholsky, Klabund, Erich Mühsam. Cette proposition réveille en elle un appétit de scène longtemps refoulé, qu'elle assouvit partiellement en compagnie du cabaret ambulant Die Leid-Artikler, dirigé par Dietrich Kittner, dans une cave du Reeperbahn à Hambourg en novembre 1963.

De retour à Berlin, c'est aussi chez Elwert \& Meurer qu'elle fait la connaissance en 1965 d'un personnage qui va changer le cours de son existence et susciter réellement sa vocation de comédienne et de cabarettiste : Paul Vasil, jeune et brillant metteur en scène français, installé depuis peu à Berlin dans le cadre de la coopération culturelle franco-allemande. C'est précisément là que commence une carrière artistique évoquée ci-après par le compagnon d'Ortrud Beginnen, James Lyons.

5 O. BEGINNEN, op. cit., p. 148 ( « Schon der Buchtitel Guck mal, schielt ja! ist die reinste Koketterie, weil ich meine Augen inzwischen sehr wirkungsvoll einsetzen kann. »). 


\section{Du livre à la scène}

Le récit d'Ortrud Beginnen n'est ni le « récit d'une carrière » ni « comment, malgré tout, j’y suis arrivée ${ }^{6}$. Un tel écrit à vocation artistique ou esthétique aurait été une faute de style, voire de goût, vis-à-vis de l'histoire de ses aïeules :

Nous, les Duncker/Böttcher/Beginnen, avons toujours été marquées du sceau de l'adversité, du tourment et de la confusion - et je n'imagine pas une seconde l'éventualité de faillir sans scrupule à la tradition. Les eaux profondes, les gargotes allemandes, une certaine insouciance [...] et un irrésistible amour de la musique, ont toujours joué chez nous un rôle décisif.

Ortrud Beginnen a toujours gardé la tête hors de l'eau, fût-ce au prix de difficultés majeures et répétées. Elle souligne avec une intensité convaincante l'influence indéniable exercée par ce passé familial sur toute sa carrière. Cette artiste s'est constamment « battue en dehors des sentiers communs », traçant son propre chemin.

Le roman familial d'Ortrud Beginnen se lit comme un roman de formation contre-culturel. Pour paraphraser les études de l'École de Francfort sur la «personnalité autoritaire » dans les années trente-quarante, on pourrait parler paradoxalement de roman de formation autoritaire d'une «personnalité antiautoritaire ». Sans s'inscrire dans une socialité de groupe qui viendra avec ses activités de chansonnière, la biographie d'Ortrud Beginnen montre également in concreto la déviance sociale d'une personnalité que toute son expérience familiale et sociale a poussé à se forger contre le système de valeurs dominant, y compris dans un sens qu'on pourrait nommer après coup « infraféministe ». Que cette déviance ait pu symboliquement investir le titre de son autobiographie de «loucheuse » manifeste peut-être inconsciemment tout ce que cette figure peu avoir d'emblématique pour sa vie autant que pour sa pratique artistique, en particulier dans son goût scénique pour la dissonance.

6 O. BEGINNEN, op. cit., p. 173 (« Bericht einer Karriere »; « wie ich es dennoch geschafft habe $)$ ).

7 O. BEGINNEN, op. cit., p. 173 ( « Wir Duncker/Böttcher/Beginnen waren schließlich geprägt durch Mißstände, Umstände und Schwierigkeiten. Und ich gedenke nicht, diese Tradition ohne weiteres zu brechen. Das tiefe Wasser, die Garküchen Deutschlands, eine gewisse Leichtlebigkeit, [...] und eine unerwiderte Liebe zur Musik haben bei uns immer eine entscheidende Rolle gespielt. »)

8 Theodor W. ADORNO, Else FRENKEL-BRUNSWIK, Daniel LEVINSON and Nevitt SANFORD, The Authoritarian Personality, Studies in Prejudice Series, Volume 1. New York: Harper \& Row, 1950. Selon les théories développées, entre autres, par Adorno, la culture sociale dominante sous 1'Empire puis Weimar et a fortiori sous le nazisme a donné maint exemple de ce type de «personnalité autoritaire » qui annonce chez certains individus un penchant pour des comportements fascistes et anti-démocratiques, de grande intolérance face aux minorités. 


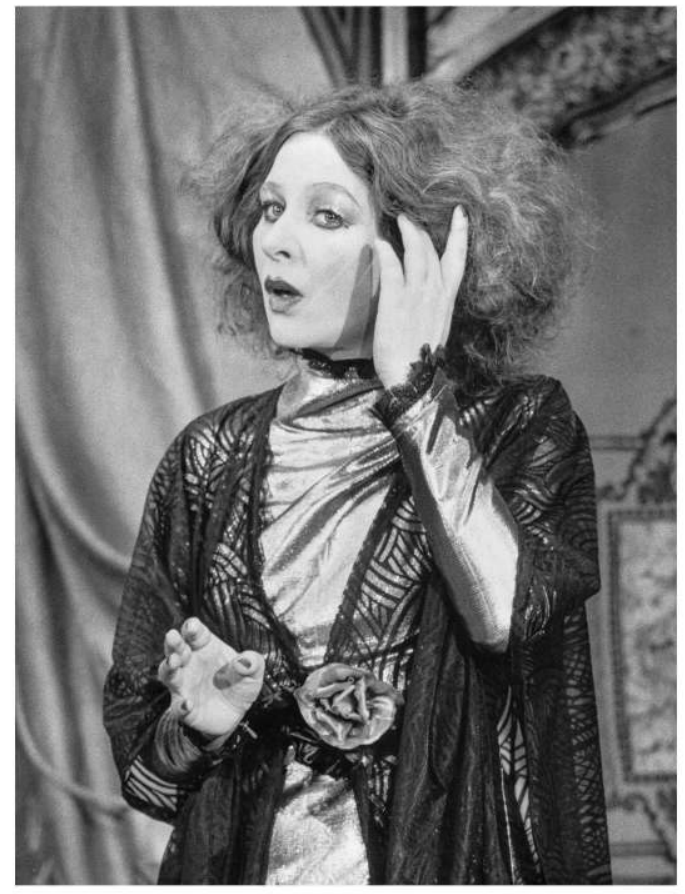

La Duse de la Ludwigkirchplatz : mimer/miner l'imago de la Diva. Letzte Rose, spectacle solo de Ortrud Beginnen au Reichskabarett, Berlin (1973). Stadtmuseum Berlin, (C) Ludwig Binder. 